Reward sensitivity following boredom and cognitive effort: A high-powered neurophysiological investigation

Marina Milyavskaya

Carleton University

Michael Inzlicht

University of Toronto

Travis Johnson

Michael J. Larson

Brigham Young University

This manuscript has been accepted for publication in Neuropsychologia. Please note that some changes may occur during the copy-editing process, such that the final published version may differ somewhat from this version.

Corresponding author: Marina Milyavskaya, marina.milyavskaya@carleton.ca

\title{
Acknowledgments
}

This research was supported by funding from the Brigham Young University College of Family, Home, and Social Sciences to M. Larson. M. Inzlicht was funded by the Social Sciences and Humanities Research Council of Canada (SSHRC; grant \#435-2014-0556). 


\begin{abstract}
What do people feel like doing after they have exerted cognitive effort or are bored? Here, we empirically test whether people are drawn to rewards (at the neural level) following cognitive effort and boredom. This elucidates the experiences and consequences of engaging in cognitive effort, and compares it to the consequences of experiencing boredom, an affective state with predicted similar motivational consequences. Event-related potentials were recorded after participants $(\mathrm{N}=243)$ were randomized into one of three conditions - boredom (passively observing strings of numbers), cognitive effort (adding 3 to each digit of a four-digit number), or control. In the subsequent task, we focused on the feedback negativity (FN) to assess the brain's immediate response to the presence or absence of reward. Phenomenologically, participants in the boredom condition reported more fatigue than those in the cognitive effort condition, despite reporting exerting less effort. Results suggest participants in the boredom condition exhibited larger FN amplitude than participants in the control condition, while the cognitive effort condition was neither different from boredom nor control. The neural and methodological implications for ego depletion research, including issues of replicability, are discussed.
\end{abstract}

Key words: rewards; cognitive effort; boredom; evoked potentials; feedback negativity; selfcontrol; ego depletion 


\section{Reward sensitivity following boredom and cognitive effort: A high-powered neurophysiological investigation}

Imagine you just spent the morning grading exams for a large course. It was boring work, and you feel drained. What do you feel like doing? Relaxation might immediately come to mind, but what if you have to get back to work? You may find other ways of rewarding yourself perhaps eating a chocolate bar, allowing yourself a few minutes to peruse Facebook, or generally engaging in some other activity that you find pleasurable or rewarding. Although people generally seek out rewarding experiences, here we wonder if this is especially the case after being bored or engaging in cognitive effort. In the present study, we empirically test whether people are more drawn to rewards after boredom or after engaging in cognitive effort compared to when they are neither, by examining the sensitivity to rewards on a neural level.

\section{Cognitive effort, fatigue, and shifting priorities towards rewards}

Neurocognitive accounts of cognitive effort view it as the mobilization of resources necessary to attain a desired level of performance (Shenhav et al., 2017). The term cognitive effort is frequently used interchangeably with cognitive control, with some suggesting that cognitive effort drives the decision to engage control (Westbrook \& Braver, 2015). Here, we define cognitive effort as the intensification of control, usually brought about by highly demanding or difficult tasks. Cognitive effort, and in particular decisions about engaging effort, is thought to be neurally mediated by the dorsal anterior cingulate cortex (dACC) and the lateral prefrontal cortex (IPFC; see Shenhav et al., 2017, for review). Importantly, cognitive effort is generally considered inherently aversive or costly (Kool, McGuire, Rosen, \& Botvinick, 2010; Westbrook, Kester \& Braver, 2013; but, see Inzlicht, Shenhav, \& Olivola, in press). According to some recent models, this cost is then weighed against the benefits of exerting the effort. 
Although there are some differences in the accounts of why mental effort is costly (see Shenhav et al., 2017, for a review), one proposed suggestion relates to the notion of opportunity costs (Kurzban, Duckworth, Kable, \& Myers, 2013). When engaging in cognitive effort on any given task, a person foregoes opportunities to engage in other (potentially valuable) tasks - these lost opportunities are termed the opportunity cost of persisting at an effortful task. According to this model, the costs of engaging in further effort are expected to rise as more effort is exerted, with the value of effort exertion (i.e., the cost-benefit ratio) diminishing proportionally (Kurzban et al., 2013). After incurring large effort costs, people may want a (proportionally large) reward. The consequences of exerting cognitive effort have also been the focus of a large body of literature on ego depletion. Ego depletion refers to a psychological state whereby people feel unable or unwilling to exert effort following an effortful task. It is akin to a state of mental fatigue (Inzlicht, Schmeichel, \& Macrae, 2014), whereby after engaging in an activity that requires effortful control, people perform more poorly on a second task, also requiring effortful control (Baumeister, Bratslavsky, Muraven, \& Tice, 1998). This sequential ego depletion paradigm (consisting of two sequential tasks requiring cognitive effort) has been used in hundreds of studies (see Hagger et al. 2010), although a recent pre-registered replication report did not find an effect for one specific operationalization of depletion (Hagger et al. 2016).

While the very existence and magnitude of the ego depletion effect are currently being examined (Carter \& McCullough, 2014; Hagger et al. 2016; Friese, Loschelder, Gieseler, Frankenbach, \& Inzlicht, in press), some have wondered how replication difficulties are affected by the possible mechanisms underlying ego depletion (Inzlicht et al. 2014; Kurzban et al., 2013). One explanation for the "depletion" period frequently observed after a demanding self-control task centers on motivation - after exerting effort on one task, people are no longer motivated to 
exert further effort on a subsequent task, and instead prefer to 'indulge' in an immediate temptation (e.g., of slacking off, venting one's anger, eating the delicious food, etc.). According to this shifting priorities model of self-control (Inzlicht et al. 2014; Milyavskaya \& Inzlicht, 2017), this shift in motivation would be accompanied by shifts in attention, perception, emotion, and memory. Thus after an effortful task, people may be more drawn to rewards, and may more readily notice opportunities to indulge in rewarding behaviours. Fatigue is thought to have similar motivational consequences (Hockey, 2013; but, see Gergelyfi et al., 2015). Indeed, some have argued that fatigue and depletion refer to the same phenomenon (Inzlicht \& Berkman, 2015), and in the present manuscript we use fatigue and depletion interchangeably.

For example, participants are more likely to gamble (Bruyneel, Dewitte, Franses, \& Dekimpe, 2009), shop (Vohs \& Faber, 2007), eat (Vohs \& Heatherton, 2000), and smoke (Shmueli \& Prochaska, 2009) after engaging in cognitive effort. In another study, exerting cognitive control for an extended period led participants to prefer smaller yet immediate monetary rewards instead of larger delayed rewards; this preference in immediate rewards was also linked to a reduced activation of the lateral prefrontal cortex (LPFC; Blain, Hollard, \& Pessiglione, 2016). Similarly, an experience-sampling study found that people were more likely to succumb to tempting desires when fatigued from repeated use of self-control throughout the day (Hofmann, Vohs, \& Baumeister, 2012). However, since enactment of a temptation is jointly determined both by the strength of the desire and the amount of effort exerted (Hofmann et al., 2012), such effects could occur either because participants are more sensitive/tempted by the rewards (i.e., the desire is greater; Schmeichel, Harmon-Jones, \& Harmon-Jones, 2010), or because they exert less self-control when faced with the desire. 
To our knowledge, only a few studies have examined the effects of ego depletion or the exertion of cognitive effort on the perception of rewards. In one study, participants who were depleted were more accurate in detecting a reward-related symbol $(\$)$ than non-reward symbol (\&) in rapidly presented images (Schmeichel et al., 2010). Using a much longer induction of cognitive fatigue (over 6 hours of cognitive control tasks) and a delay discounting task, Blain and colleagues (2016) found an increase in preference towards more immediate monetary rewards (instead of delayed but larger rewards). In another particularly relevant study, dieters who were depleted exhibited greater food-cue-related activity in areas of the brain associated with coding reward values, and other areas associated with self-control (Wagner, Altman, Boswell, Kelley \& Heatherton, 2013). In that study, chronic dieters either completed a depletion task or a control task and then viewed desirable foods while in an fMRI scanner. Participants in the depletion condition had greater activation in the orbitofrontal cortex, as well as lesser functional connectivity between the orbitofrontal cortex and the inferior frontal gyrus, than those in the control condition (Wagner et al., 2013). Together, these studies support the possibility that after people exert cognitive effort, they exhibit increased sensitivity towards rewards.

\section{Boredom as similar motivational state}

If the consequences of effort expenditure affect states of motivation, then depletion might have similar motivational properties as other states that influence motivation towards rewards. One such state is boredom. Boredom is typically described as an affective state that results from the inability to "successfully engage attention with internal or external information" (Eastwood, Frischen, Fenske, \& Smilek, 2012, pg. 484); it is characterized by "core motivational deficits accompanied by a phenomenological experience of a lack of interest or affective engagement." (Goldberg, Eastwood, Laguardia \& Danckert, 2011, pg. 649). Here, we similarly define boredom 
as a state produced by under-stimulation, where desires for stimulation and engagement are not being met.

Although it may at first seem paradoxical that engaging in cognitive effort would lead to the same consequences as boredom, there are several reasons to theorize that boredom might have similar motivational consequences to depletion. First, research on vigilance tasks that require participants to monitor displays for infrequent stimuli for a prolonged period of time (e.g., Mackworth, 1948) have been alternatively interpreted as inducing fatigue (i.e., depletion) or boredom (Pattyn, Neyt, Henderickx, \& Soetens, 2008). Indeed, research has shown that both the "depletion of information-processing resources" (i.e., an overload of the attentional system; Pattyn et al. 2008, pg. 377) and under-arousal both lead to a decrease in vigilance (see Pattyn et al. 2008). In other words, vigilance tasks might induce both (1) fatigue and (2) boredom, both of which might have similar downstream consequences on subsequent behavior.

Second, animal models of boredom find that animals housed in cages with no opportunities for enrichment display more interest in novel stimuli and consume more food rewards (Meagher \& Mason, 2012). That is, these 'bored' animals are more attuned to rewards. Similarly, in humans greater and more frequent experiences of boredom have been linked to engaging in more impulsive, reward-seeking behaviour including gambling (Blaszczynski et al. 1990), overeating and binge eating (Abramson \& Stinson, 1977; Myhre et al. 2015), and alcohol and drug abuse (Iso-Ahola \& Crowley, 1991); this is similar to the impulsive tendencies of depleted participants (e.g., Vohs \& Heatherton, 2000; Vohs \& Faber, 2007). While research finds that people who are generally prone to boredom engage more frequently in such impulsive behaviours, to our knowledge there has not been any research examining whether state boredom would directly affect a person's orientation towards rewards. 
One proposed function of boredom is that boredom serves as an indicator to pursue an alternate goal (Bench \& Lench, 2013). A similar motivational function of depletion has also been proposed, with depletion or fatigue seen as a stop-signal, a signal to end cognitive effort and engage in other pursuits (Hockey, 2013; Inzlicht et al., 2014; Kurzban et al. 2013). This suggests that boredom and depletion might have similar functions in orienting humans to disengage from current behaviour and seek other (more rewarding) alternatives. A purpose of the current study, then, is to test whether boredom also elicits a stronger orientation towards rewards. Specifically, we predicted that participants who were either bored or depleted would show an increased sensitivity to rewards compared to participants who were neither depleted nor bored.

\section{The Feedback Related Negativity (FN)}

In the present study, we looked at reward sensitivity as the brain's immediate responses to reward using electroencephalographic (EEG) recordings, focusing on the feedback negativity (FN) component of the scalp-recorded event-related potential (ERP). The FN is a negative deflection in the ERP at frontocentral electrode recording sites that occurs around $250 \mathrm{~ms}$ after feedback presentation and is larger (i.e., more negative) to unfavorable than favorable outcomes. Recent research (see Proudfit, 2015 for review) suggests that the FN may represent a positive response to positive feedback (reflecting gains or rewards) that is reduced following negative feedback, with the difference between negative and positive feedback (negative minus positive) representing the negative deflection seen in the FN. In this view, the FN is actually a decrease in the positivity associated with reward/favorable feedback (Proudfit, 2015), rather than a negative deflection per se. Nonetheless, The FN has good psychometric properties (Levinson et al., 2017) and has been correlated with subjective interest in rewards (Bress \& Hajcak, 2013), and with approach motivation more generally (Threadgill \& Gable, 2016). In the present study, we 
examine the FN to evaluate the extent to which depleted and bored participants are drawn towards rewards.

\section{Present study}

We conducted a high-powered study to examine the effects of effort expenditure and boredom on reward sensitivity. We also included measures of phenomenology as a manipulation check, expecting that the effort condition would result in high self-reported effort and fatigue (compared to the boredom condition), and that the boredom condition would lead to greater boredom (compared to the effort condition). For our main research questions, we first hypothesized that participants who exert cognitive effort (i.e., the effort condition) would have a greater sensitivity to rewards (as indexed by the FN) than participants in the control condition. Importantly, we expected the boredom condition to have an effect similar to the effort condition, with both resulting in higher sensitivity to rewards than the control condition. We also conducted exploratory analyses to examine whether the type of reward mattered. Specifically, previous research has found that monetary rewards elicited greater FN than no-value rewards; while we expected to replicate this main effect, we did not have specific predictions as to whether there would be an interaction with condition.

\section{Method}

\section{Participants}

There is no consensus on the typical effect size for the sequential task paradigm (although the effect is likely small, if it exists at all; see Inzlicht, Gervais \& Berkman, 2015); more importantly, since we used a neural index of sensitivity to rewards rather than a measure of

control, we had nothing to guide our selection of expected effect size. We thus aimed for a medium effect. A power analysis indicated that 210 participants would allow the detection of a 
medium effect $(f=.25)$ in a one-way ANOVA with 3 groups with a power of .90 (G*Power). We set out to recruit at least 210 participants, but continued recruiting until the end of the academic semester. Participants were 243 university students who completed the study for course credit a sample that is an order of magnitude larger than typical EEG studies. Thirty-one participants were excluded from the experiment due to equipment malfunction or too much noise in the data, resulting in unusable EEG data (21 participants), or insufficient artifact-free trials (10 participants; see explanation below). The final sample consisted of 212 participants (41.9\%female) ages $18-26(\mathrm{M}=20.84, \mathrm{SD}=2.09)$. All participants were healthy, free from neurological disease, and had no food allergies.

\section{Procedure}

Participants came into the lab and were connected to the EEG system. They were then randomly assigned to one of three conditions, completing either one of two numbers tasks (effort and boredom conditions) followed by the computerized door task (designed to elicit the FN), or, in the neutral condition, went straight to the computerized doors task. For participants in the effort and boredom conditions, the computerized door task was presented as a separate study: "Since you are already connected to the EEG, and our study is not very long, we have partnered with another researcher to help them pilot a new task, so we ask that you do this task now." After the door task, participants completed personality questionnaires (included in the study for exploratory purposes; see https://osf.io/e39as/ for all materials). Participants in the control condition completed the same numbers task as those in the effort condition after all other study procedures were completed (to equate participation length, as required by the institution's IRB board). At the end of the study, participants were given their additional monetary and candy reward and debriefed. The outline of the procedure is illustrated in Figure 1. 


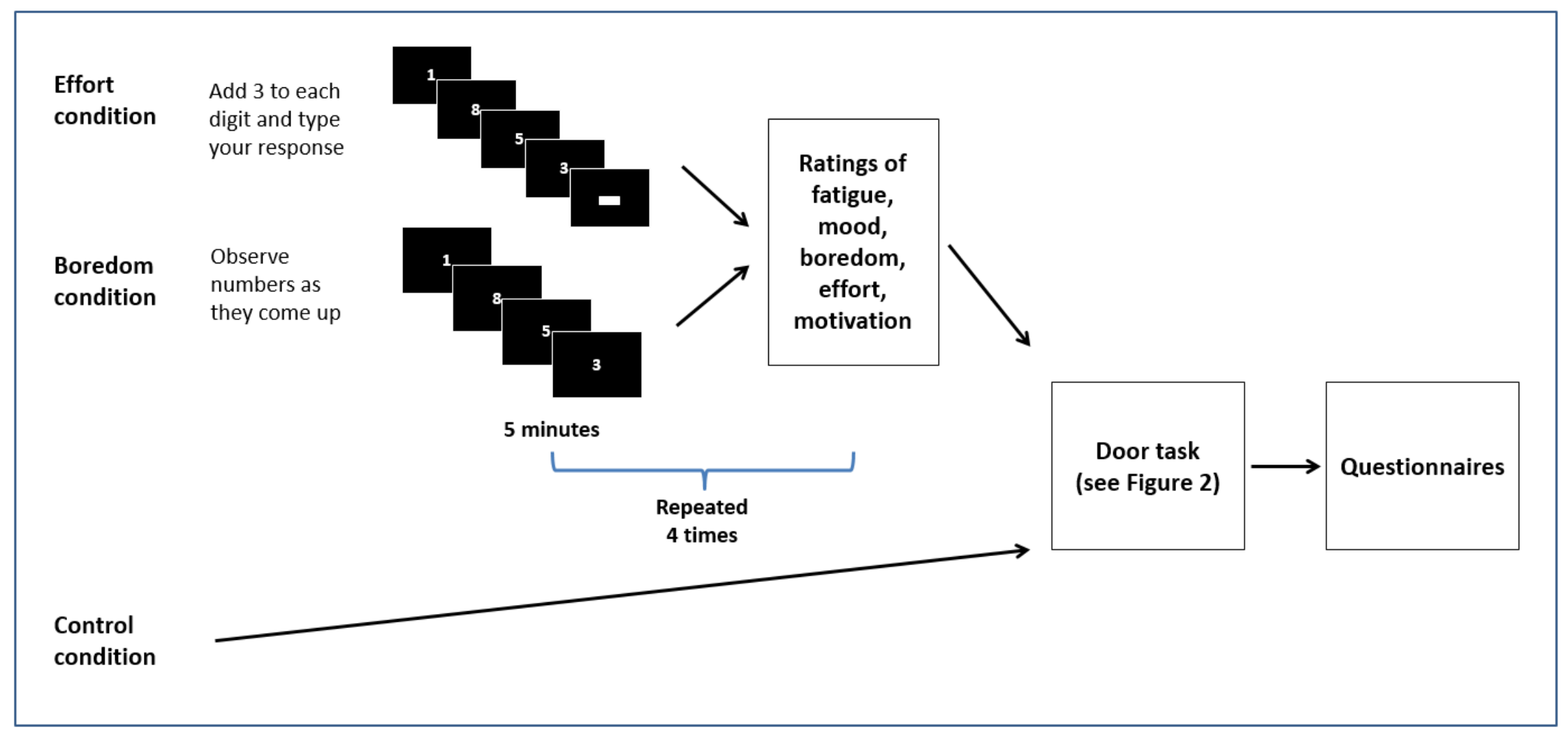

Figure 1. The procession of experimental tasks across the three conditions. Participants in the effort and boredom conditions completed 20 minutes of either the add 3 task or the passive number viewing task in four blocks of five minutes each; each block was followed by self-reported ratings. Participants in all three conditions then proceeded to the door task followed by questionnaires. 


\section{Experimental manipulation}

Participants were randomly assigned using a random number generator into one of three conditions. In the effort and boredom conditions, participants completed a 20-minute task involving either number manipulation or passive number viewing. In both tasks, participants were presented with strings of four digits one at a time. In the effort condition, participants were asked to add 3 to each digit and type their response (e.g., 9234 results in 2567; Kahneman, 2011). This is a task that requires high cognitive effort, result in maximal pupil dilation, and is experienced as subjectively difficult (Kahneman, 2011). Participants' accuracy improved over time (across the 4 blocks) - in a within-person repeated-measures ANOVA, there was a linear effect of time, $\mathrm{F}(1,85)=32.39, \mathrm{p}<.001$, with mean accuracy ranging from .52 in block 1 to .66 in block 4. Participants were not provided with any feedback during the task. In the boredom condition, participants were asked to passively observe the numbers as they came up. They were not instructed to actively inhibit anything, and there were not instructed to maintain any goal active in working memory, such that the participants could have mind-wandered the entire time if they wished to do so. Since no active effort is required other than simple vigilance, we expected that participants would experience a lack of engagement and stimulation, resulting in under-arousal characteristic of boredom. Both tasks consisted of 4 blocks of 5 minutes each, for a total of 20 minutes. There were 150 trials per block in the boredom condition, and participants completed between 129 and 187 trials per block in the effort condition (depending on how quickly they entered their responses into the system). After each block, participants were asked to rate their fatigue, mood, boredom, effort, and motivational locus (external vs. internal) using a visual-analog scale (VAS) for each item: "How are you feeling right now” (Fatigued-Energized); "What is your mood right now" (Unpleasant- Pleasant); "How are you feeling right now?" 
(Bored-Interested); "How hard are you trying to do well on the task" (Not trying very hard trying very hard); "How much do you feel like you are participating in this study" (Because I feel like I have to - Because it is personally important to me). The VAS scales were translated into a number from 0 to 100 , with larger numbers representing the labels on the right.

\section{Computerized door task}

All participants then completed the door lottery task. This task was modeled on a similar task commonly used to elicit the FN (Proudfit, 2015; Weinberg, Riesel \& Proudfit, 2014), but with one important difference: we added a third reward option consisting of a food reward (M\&M candy). Since food, and in particular sugar/glucose, has been conceptualized by both lay people and some researchers as an effective antidote to depletion (Baumeister \& Tierney, 2011; Gailliot et al., 2007; cf. Kurzban, 2010), we were interested in whether these lay theories would translate into people being more oriented towards such food-related rewards, such that M\&M candy would be especially rewarding to someone who has recently exerted effort.

The door lottery task we used consisted of 144 trials organized in 4 blocks to provide participants with breaks (see Figure 2). On each trial, participants saw two doors and were asked to choose one to open. Once the door was opened, participants received feedback to indicate whether they won (green arrow pointing up) or lost (red arrow pointing down). Prior to each trial, participants were informed whether on the upcoming trial they would have the chance to earn or lose money, candy (M\&Ms), or nothing. There were 48 trials for each type of reward (randomized within-block), with positive feedback presented on $50 \%$ of the trials. Prior to the task, participants were informed that they would receive 50c for each 'correct' money trial and 2 M\&M candies for each 'correct' candy trial, and lose $25 \mathrm{c}$ on each 'incorrect' money trial or 1 M\&M candy on each incorrect' candy trial (due to losses looming larger than gains; see Proudfit, 
2015). The order and timing of all stimuli are as follows: (i) one of three texts ('Click for the next round', 'Click for a chance to win money' or 'Click for a chance to win candy') appeared until the response is made; (ii) the graphic of two doors is presented until a response is made, (iii) a fixation mark is presented for $1000 \mathrm{~ms}$, (iii) a feedback arrow is presented for $2000 \mathrm{ms,}$ (iv) a fixation mark is presented for $1500 \mathrm{~ms}$. In between each block, participants were told how much money and M\&Ms they earned in the previous block (this was constant for all participants). The task lasted approximately 15-20 minutes (depending on the speed of participants' responses). There were no differences in reaction time on the doors task across conditions $(F(2,231)=.953, p=.387)$, and a small correlation between the reaction time and the $\mathrm{FN}(r=-.16, p=.026)$, such that those who are faster to respond on the doors task have a slightly larger FN response (i.e., those who are more sensitive to reward are slightly quicker/more impulsive). 


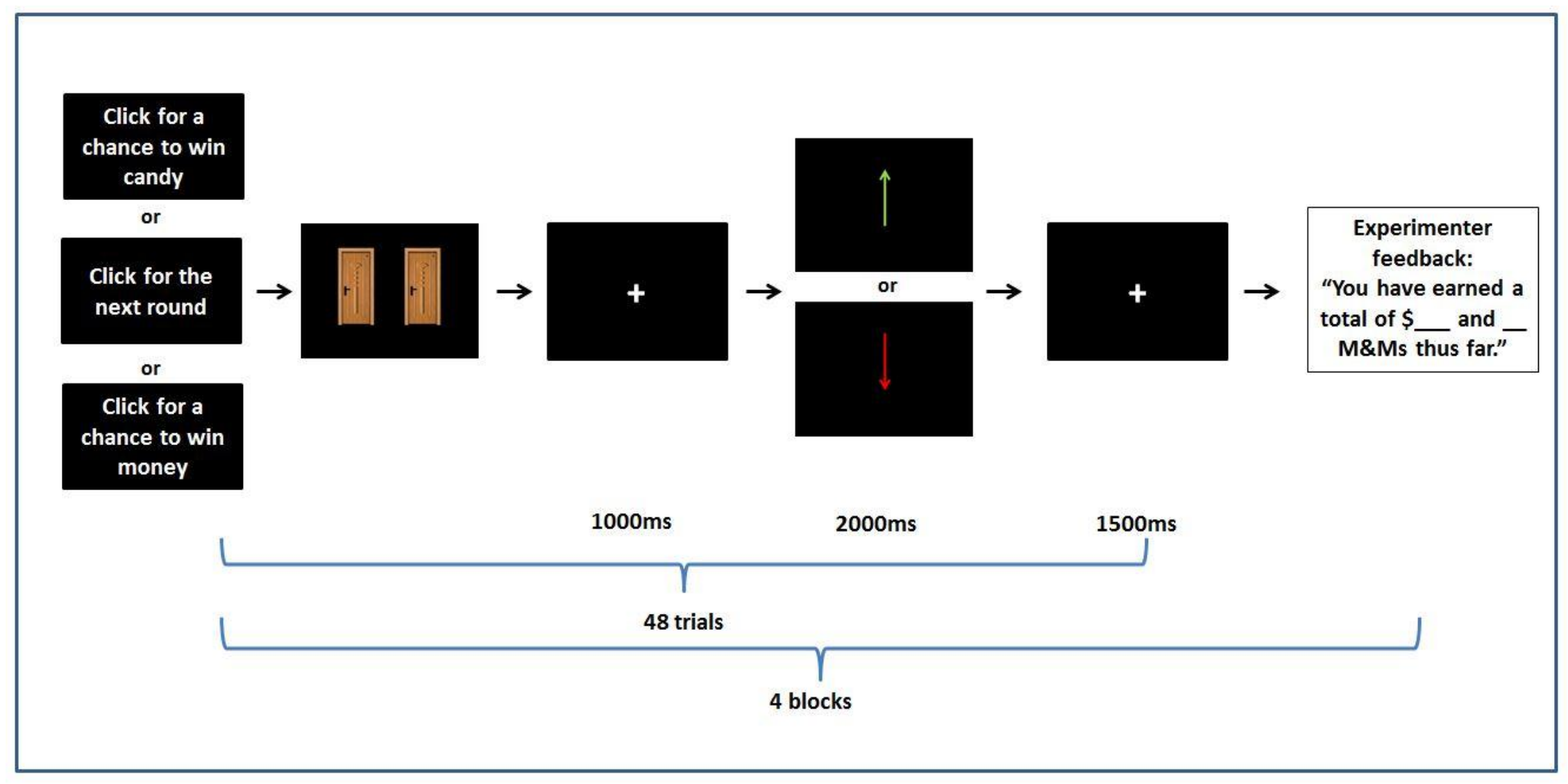

Figure 2. The computerized door task. Participants completed four blocks of the door task, each consisting of 36 trials. Before each trial participants were informed whether on the upcoming trial they would have the chance to earn or lose money, candy (M\&Ms), or nothing. Participants then saw two doors and were asked to choose one to open. Once the door was opened, participants received feedback to indicate whether they won (upwards green arrow) or lost (downward red arrow); positive feedback was presented on 50\% of the trials. Participants received 50c or $2 \mathrm{M} \& \mathrm{M}$ candies for each 'correct' trial, and lost 25c or $1 \mathrm{M} \& \mathrm{M}$ candy on each 'incorrect' trial. EEG signals were time-locked to the presentation of the feedback (upwards green arrow or downward red arrow). 


\section{EEG Data Acquisition and Reduction.}

Electroencephalogram data was recorded during the doors task from 128 scalp sites using a geodesic sensor net and Electrical Geodesics, Inc., (EGI; Eugene, Oregon) amplifier system (20K gain, nominal bandpass $=.10-100 \mathrm{~Hz})$. Electrode placements enabled recording vertical and horizontal eye movements reflecting electro-oculographic (EOG) activity. Data from the EEG was referenced to $\mathrm{Cz}$ and digitized continuously at $250 \mathrm{~Hz}$ with a 16-bit analog-to-digital converter. A right posterior electrode approximately two inches behind the right mastoid served as common ground. Electrode impedance was maintained below $50 \mathrm{k} \Omega$. EEG signals were timelocked to the presentation of the feedback (upwards green arrow or downward red arrow). EEG data was segmented off-line into epochs between -200 ms before and $800 \mathrm{~ms}$ after stimulus presentation with a $200 \mathrm{~ms}$ baseline correction. Data were filtered with 0.1 to $30 \mathrm{~Hz}$ bandpass. We removed eye blinks and saccades using independent components analysis (ICA) implemented in the ERP PCA Toolkit (Dien, 2010). The ICA components that correlated at 9 with the scalp topography of two templates, one generated based on the current data and another provided by the ERP PCA Toolkit author, were removed. Trials were considered bad if more than $15 \%$ of channels were marked bad. Channels were marked bad if the fast average amplitude exceeded $100 \mu \mathrm{V}$ or if the differential average amplitude exceeded $50 \mu \mathrm{V}$.

Previous work with high-density arrays suggests averaging across electrodes provides increased reliability (Baldwin et al. 2015). Thus, from the artifact-free epochs we extracted the ERPs within the time window from $175 \mathrm{~ms}$ to $225 \mathrm{~ms}$ across an average of 7 frontal electrodes including electrodes 5, 6 (FCz), 7, 12, 13, 106, and 112 (see Larson et al. 2010 for figure with electrode locations). This time window was selected using the collapsed localizers approach 
suggested by Luck and Gaspelin (2016) to deal with the problem of multiple implicit comparisons inherent in ERP research.

In the collapsed localizer approach for this study, we averaged all the waveforms across all conditions and selected the time window that demonstrated the largest negative deflection at the general time frame of interest. This ensured that we did not select a window based on visual differences between conditions (see Luck \& Gaspelin, 2016). To obtain the general FN, the average of all 'correct' feedback trials was subtracted from the average of all incorrect feedback trials (irrespective of reward type). More negative numbers indicate a stronger FN. Then, to obtain the FN for each specific reward type (for use in multilevel analyses to examine differences by type of reward) the average of all 'correct' feedback trials for each given reward type was subtracted from the average of all incorrect feedback trials for that reward type. For these, only instances with 10 or more artifact-free trials of that type were used, such that some participants did not have data for one or two of the reward types.

ERP amplitude estimates were determined to be reliable with a minimum of 10 trials per condition (see Clayson et al. 2013). As reliability is dependent on each specific sample and study), dependability estimates (a generalizability theory [G-theory] analogue of reliability) were calculated for each group and condition. Using formulas provided by Baldwin et al. (2015) in the ERP Reliability Analysis (ERA) Toolbox v 0.3.2 (Clayson \& Miller, in press). The ERA Toolbox calculated ERP dependability based on algorithms from generalizability theory (see Baldwin et al., 2015 for review) and used CmdStan v 2.10.0 to implement the analyses in Stan. When each condition had at least 10 trials, point dependability estimates all exceeded .70 except $1($ Effort Neutral Correct trials $=.67)$. Dependability estimates, $95 \%$ credible intervals, mean numbers of trials, and trial count range as a function of group and condition are presented in 
Table 1. These findings do not contradict those of Levinson et al., (2017) who suggest a minimum of 20 trials is needed for a reliable $\mathrm{FN}$ because reliability/dependability are specific to each individual sample and this particular sample simply required fewer trials to reach dependability than the Levinson study.

Table 1. Dependability estimates for each group and condition.

\begin{tabular}{lcccc}
\hline & Dependability & $95 \%$ CI & Mean \# Trials & Trial Range \\
\hline Boredom Candy Correct & .85 & $.80 ; .90$ & 20.2 & $12 ; 24$ \\
Boredom Candy Incorrect & .83 & $.77 ; .89$ & 20.1 & $13 ; 24$ \\
Boredom Money Correct & .91 & $.87 ; .94$ & 20.6 & $11 ; 24$ \\
Boredom Money Incorrect & .88 & $.83 ; .92$ & 19.9 & $11 ; 24$ \\
Boredom Neutral Correct & .76 & $.66 ; .84$ & 20.4 & $12 ; 24$ \\
Boredom Neutral Incorrect & .80 & $.71 ; .87$ & 20.1 & $11 ; 24$ \\
Effort Candy Correct & .83 & $.77 ; .88$ & 20.1 & $11 ; 24$ \\
Effort Candy Incorrect & .80 & $.73 ; .87$ & 19.8 & $10 ; 24$ \\
Effort Money Correct & .90 & $.86 ; .93$ & 20.3 & $12 ; 24$ \\
Effort Money Incorrect & .85 & $.79 ; .90$ & 19.8 & $12 ; 24$ \\
Effort Neutral Correct & .67 & $.54 ; .77$ & 20.1 & $14 ; 24$ \\
Effort Neutral Incorrect & .79 & $.71 ; .85$ & 20.2 & $10 ; 24$ \\
Control Candy Correct & .82 & $.75 ; .87$ & 20.8 & $12 ; 24$ \\
Control Candy Incorrect & .78 & $.70 ; .85$ & 20.7 & $12 ; 24$ \\
Control Money Correct & .84 & $.78 ; .89$ & 21.0 & $11 ; 24$ \\
Control Money Incorrect & .85 & $.80 ; .90$ & 20.5 & $13 ; 24$ \\
Control Neutral Correct & .81 & $.75 ; .87$ & 20.8 & $12 ; 24$ \\
Control Neutral Incorrect & .74 & $.63 ; .82$ & 20.6 & $12 ; 24$ \\
\hline & & & & \\
\hline
\end{tabular}




\section{Results}

Syntax and output for all results, as well as the data, are posted on OSF (https://osf.io/e39as/).

\section{Preliminary analyses: phenomenology of depletion and boredom conditions}

We first examined differences between the effort and boredom conditions in self-report ratings of fatigue, boredom, mood, and effort. A mixed (between: condition X within: block) ANOVA found that for all four variables there was a main linear effect of time, as well as a main effect of condition (see Table 2). ${ }^{1}$ There was also a significant linear time by condition interaction for fatigue and mood but not for boredom or effort (see Table 2). Figure 3 illustrates these effects. Participants reported feeling more bored (less interested) as the task went on, and boredom was stronger in the bored condition, supporting the effectiveness of our boredom manipulation. Similarly, as expected, participants in the effort condition exerted more effort throughout the task. These self-report ratings validate our method, confirming that participants exerted greater cognitive effort in the effort condition, and felt more bored in the boredom condition. Participants in the bored condition also reported a sharp decrease in mood throughout the experiment, although participants in the effort condition reported more unpleasant mood.

Surprisingly, although participants in the bored condition reported levels of fatigue similar to the effort condition after the first block, they reported more fatigue as time went on. Remaining bored, in other words, might have been phenomenologically more fatiguing than continuously exerting cognitive effort.

\footnotetext{
${ }^{1}$ We also conducted the same analysis with motivational locus (i.e., to what extent they are doing the task because they feel like they have to vs. they personally believe that it is important). There was no main effect of condition, $F(1,159)=1.36, p=.245, \eta_{2}=.008$, suggesting that boredom and effort did not have differential effects on motivation. While there was a quadratic effect for time (such that internal motivation increased then dropped, $\left.F(1,159)=9.87, p=.002, \eta_{2}=.06\right)$, there were no interactions with condition. Since this did not seem particularly relevant to the broader paper, we do not discuss it further. Full results are presented in additional documentation on OSF.
} 
Table 2. Phenomenology of depletion and boredom conditions across time.

\begin{tabular}{|c|c|c|c|c|}
\hline & $\mathrm{F}$ & $\mathrm{p}$ & $\eta_{2}$ & $90 \% \mathrm{CI}$ \\
\hline \multicolumn{5}{|c|}{ Main effect of time (linear) } \\
\hline Fatigue & 53.60 & $<.001$ & .25 & $.16 ; .34$ \\
\hline Boredom & 34.29 & $<.001$ & .18 & $.10 ; .26$ \\
\hline Mood & 31.70 & $<.001$ & .17 & $.09 ; .25$ \\
\hline Effort & 8.06 & .005 & .05 & $.01 ; .11$ \\
\hline \multicolumn{5}{|c|}{ Main effect of condition } \\
\hline Fatigue & 7.93 & .005 & .05 & $.01 ; .11$ \\
\hline Boredom & 23.99 & $<.001$ & .13 & $.06 ; .21$ \\
\hline Mood & 8.97 & .003 & .05 & $.01 ; .12$ \\
\hline Effort & 41.20 & $<.001$ & .21 & $.12 ; .29$ \\
\hline \multicolumn{5}{|c|}{ Time* condition interaction } \\
\hline Fatigue & 8.31 & .004 & .05 & $.01 ; .11$ \\
\hline Boredom & .77 & .383 & .01 & $.00 ; .04$ \\
\hline Mood & 17.65 & $<.001$ & .10 & $.04 ; .18$ \\
\hline Effort & 3.87 & .051 & .02 & $.00 ; .08$ \\
\hline
\end{tabular}

Note: $\eta_{2}$ were obtained using the SPSS calculator from Wuensch (2012). 90\% CIs are reported (instead of $95 \%$ ) because $\eta_{2}$ cannot be negative (see http://daniellakens.blogspot.ca/2014/06/calculating-confidence-intervals-for.html for a clear explanation) 


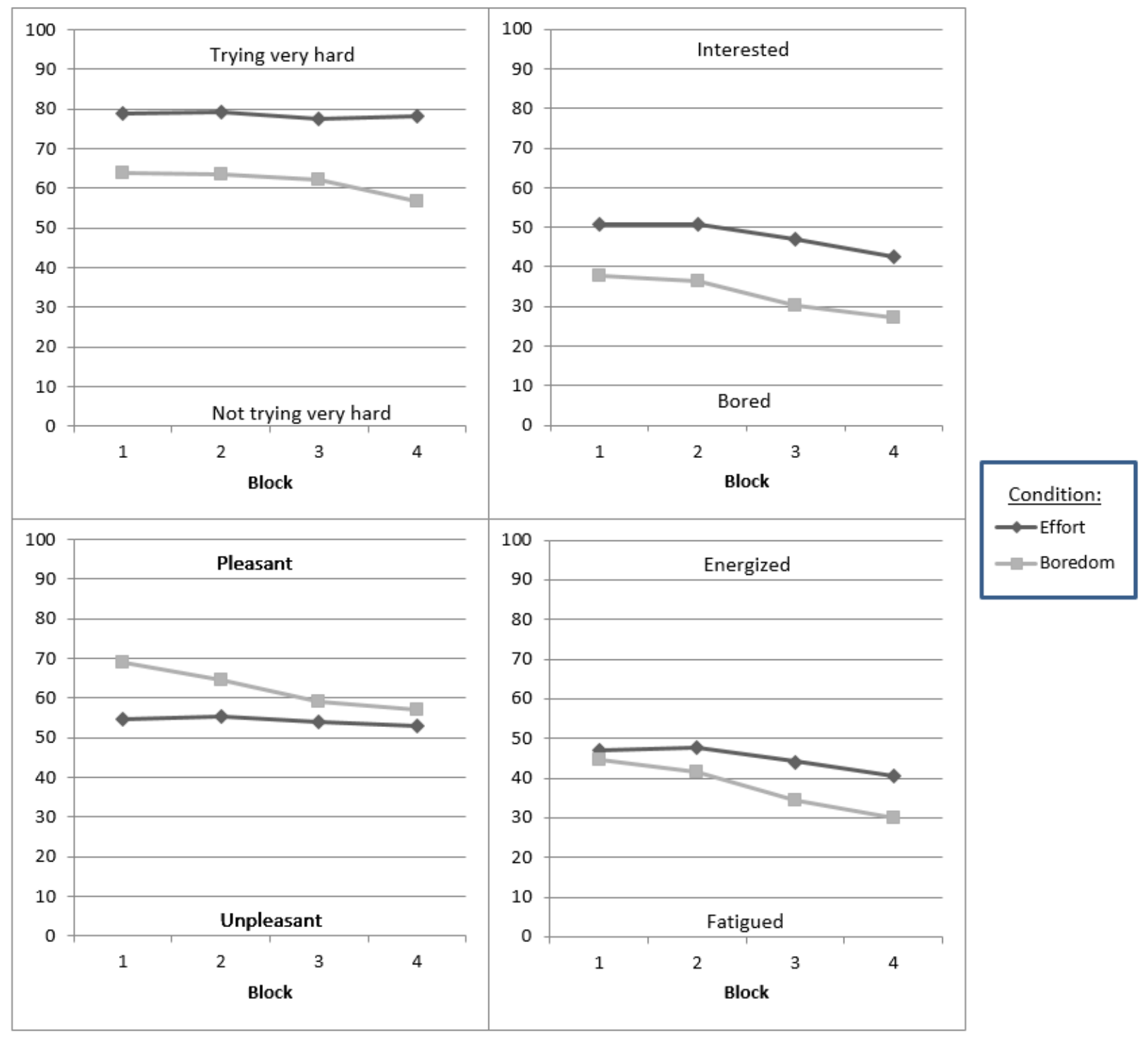

Figure 3. Phenomenology of effort and boredom conditions across blocks. Ratings were made on visual-analog scales ranging from 0-100. The wording for each construct were as follows: Effort (top left) "How hard are you trying to do well on the task" (Not trying very hard - trying very hard); Boredom (top right) "How are you feeling right now?” (Bored-Interested); Mood (bottom left) "What is your mood right now" (Unpleasant- Pleasant); Fatigue (bottom right) "How are you feeling right now" (Fatigued-Energized). 


\section{Main analyses: differences in $\mathrm{FN}$ across conditions}

We first conducted a between-subject analysis comparing the three conditions on overall FN amplitude. Table 3 reports all the means. There were significant differences among conditions, $\mathrm{F}(2,211)=3.17, \mathrm{p}=.04, \eta_{2}=.03,90 \% \mathrm{CI}[.0004 ; .0700]$, although the effect was small in magnitude. To more directly test our hypotheses, we conducted two planned contrasts: one comparing both the effort and boredom conditions to the control condition, and the other comparing the effort and boredom conditions to each other; we expected the former, but not the latter, to be different from one another. The first planned contrast showed that together, the effort and boredom conditions were significantly different from the control condition, $t(209)=-2.04, p$ $=.04, d=.30,95 \% \mathrm{CI}[.01 ; .59]$, although again the effect size was only modest in magnitude. $\mathrm{A}$ post-hoc examination of the means showed that the effects appeared to be driven by the boredom condition - that is, participants in the boredom condition had the most-negative FN among the three groups (see Table 3). The difference between the boredom and control condition was significant even after correcting the alpha level for the three comparisons (i.e., lower than .05/3, as per a Bonferroni correction), $t(135)=-2.58, p=.011, d=.44,95 \% \mathrm{CI}[.10 ; .78]^{2}$. The second planned contrast, comparing the effort and boredom condition, showed that the two were not different from one another, $\mathrm{t}(209)=1.53, \mathrm{p}=.13, \mathrm{~d}=.24,95 \%$ CI [-.09;.57]. Importantly, the direction of the means was such that the boredom condition showed the strongest FN response. Figure 4 illustrates the FN waveforms and topographical maps for each condition.

At the request of a reviewer, we also conducted additional sub-group analyses comparing only those participants who were more fatigued by the end of the last block in the effortful

\footnotetext{
${ }^{2} d$ and confidence interval are calculated based on t-test directly comparing the two conditions.
} 
condition than the average rating for the boredom condition (energy score $<=30, n=21$ ) against those who were less fatigued by the end of the boredom manipulation than the average of the effortful condition (energy score $>=40, \mathrm{n}=26$ ). Among these subgroups (and when compared to everyone in the control condition, $n=69$ ), the results were essentially the same, albeit slightly weaker (likely due to reduced statistical power), as when we used the entire population. This suggest that even among the participants for whom the manipulation "worked", results are basically unchanged. The full results can be found in the supplementary analyses section on OSF $^{3}$

Table 3. Overall FN difference amplitude

\begin{tabular}{lcccc}
\hline & $\underline{\mathrm{N}}$ & $\underline{\text { Mean }}$ & $\underline{\mathrm{SD}}$ & $\underline{95 \% \mathrm{CI}}$ \\
Effort & 75 & $-.86_{\mathrm{ab}}$ & 1.37 & $-1.17 ;-.54$ \\
Boredom & 68 & $-1.19_{\mathrm{a}}$ & 1.37 & $-1.52 ;-.86$ \\
Control & 69 & $-.64_{\mathrm{b}}$ & 1.14 & $-.91 ; .36$
\end{tabular}

Note: Values not sharing a subscript are significantly different from each other in posthoc analyses. The confidence intervals (CIs) are around the mean, and do not refer to any comparisons.

\footnotetext{
${ }^{3}$ We also conducted additional exploratory analyses on the relation between personality measures and reward sensitivity. Eighteen correlations were computed; the FN correlated only with conscientiousness $(r=-.165, p=.016)$ and with impulsiveness $(r=.167, p=.015)$. Including either conscientiousness or impulsiveness as a control in the main analyses weakened the results slightly; however, there were no interactions with either variable, suggesting that the results remain the same across all levels of both personality measures. The full output can be found on OSF under 'supplementary analyses'.
} 


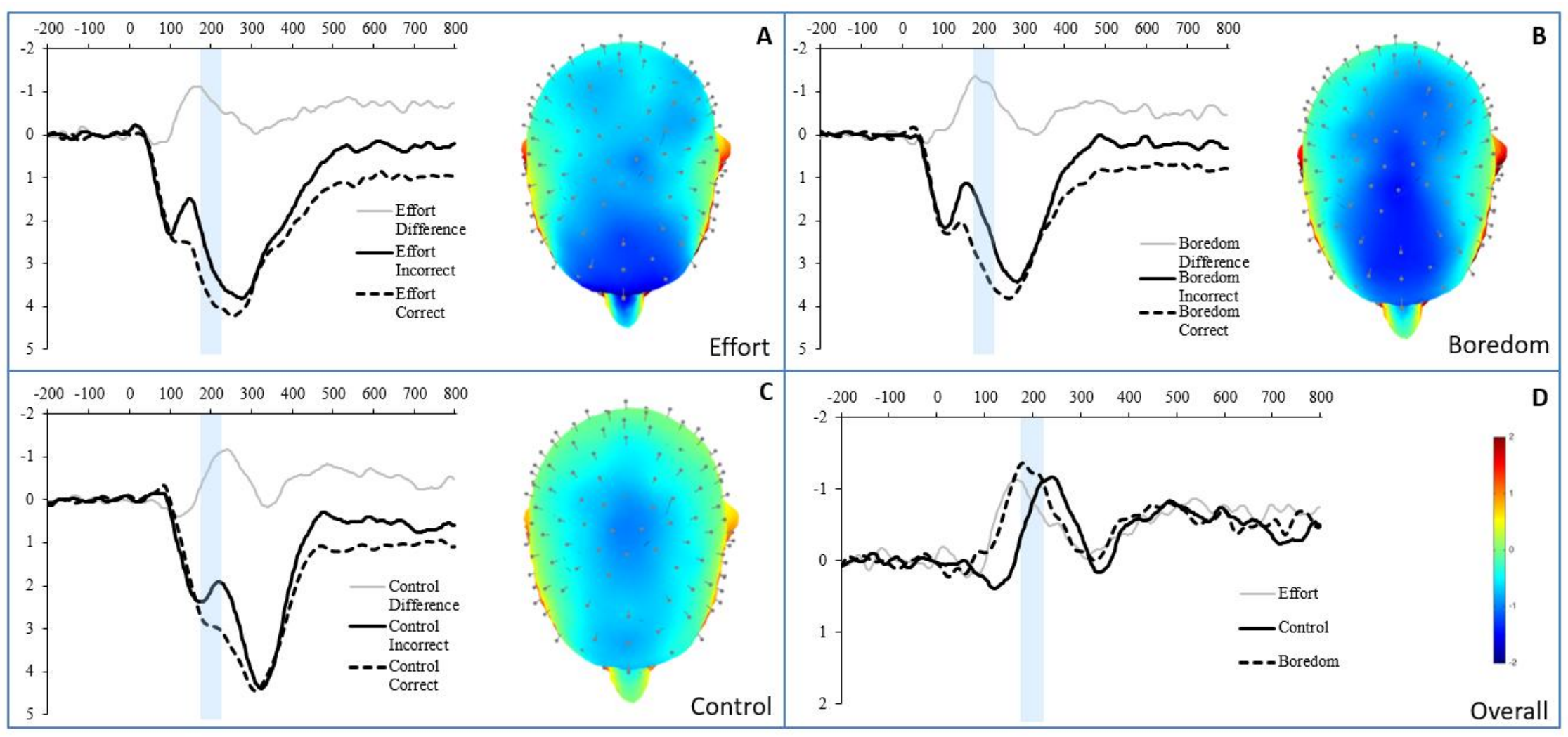

Figure 4. Response-locked ERP waveforms comparing gain (correct) and loss (incorrect) trial waveforms, in the Effort (top left), Boredom (top right), and Control (bottom left) conditions, as well as the waveforms of the difference between gains and loses across the three conditions (bottom right). For each panel, response onset occurred at $0 \mathrm{~ms}$ and negative is plotted up. The grey bar illustrates the time interval (175-225ms) used for calculating the value of the FN in the present study, which we determined with a collapsed localizer approach. Also shown are topographic maps depicting differences between response to gains and losses in Effort (top left), Boredom (top right), and Control (bottom left) conditions at the $200 \mathrm{~ms}$ time. 
To examine whether the type of reward matters, we ran a 3(between) X 3(within) MIXED model in SPSS. Condition (effort, boredom, control) was entered as a between factor, and type of reward (money, candy, positive feedback) was entered as a within factor. There was a main effect of reward type, $\mathrm{F}(2,391.60)=5.88, \mathrm{p}=.003, \eta_{2}=.03,90 \% \mathrm{CI}[.006 ; .060]$, and a marginal main effect of condition, $\mathrm{F}(2,195.89)=2.94, \mathrm{p}=.055, \eta_{2}=.03,90 \% \mathrm{CI}[.000 ; .072]$ but no interaction effect, $\mathrm{F}(4,391.58)=.70, \mathrm{p}=.593, \eta_{2}=.007,90 \% \mathrm{CI}[.000 ; .016]$. A post-hoc examination of the means showed that across the types of rewards, the boredom condition $(\mathrm{M}=-$ $1.22,95 \% \mathrm{CI}[-1.53 ;-.91])$ elicited a significantly larger $\mathrm{FN}$ that in the control condition $(\mathrm{M}=-$ $.69,95 \% \mathrm{CI}[-.99 ;-.39])$, mean difference $=.5295 \% \mathrm{CI}[.10 ; .96], \mathrm{p}=.017)$. Furthermore, across the three conditions, money elicited a larger FN response $(\mathrm{M}=-1.19,95 \% \mathrm{CI}[-1.42 ;-.95])$ than both candy $(\mathrm{M}=-.90 ; 95 \% \mathrm{CI}[-1.13 ;-.67]$; mean difference $=.2995 \% \mathrm{CI}[.01 ; .56], \mathrm{p}=.041)$ and positive feedback $(\mathrm{M}=-.71,95 \% \mathrm{CI}[-.95 ;-.48]$; mean difference $=.4895 \% \mathrm{CI}[.20 ; .75], \mathrm{p}=.001)$; the latter were not significantly different from one another, mean difference $=.1995 \% \mathrm{CI}[-$ $.09 ; .46], \mathrm{p}=.177$. However, as there was no interaction, this did not differ across conditions.

\section{Linking phenomenological reports of depletion with FN.}

To further examine whether self-reports of depletion were related to reward sensitivity, we conducted exploratory analyses testing the correlation between self-reported boredom, fatigue, and effort (averaged across the 4 time points) with reward sensitivity as indexed by the FN. Since these self-report measures were only available for participants in the boredom and effort conditions, only data from these participants $(\mathrm{N}=143)$ was used. There was no relationship between these self-report variables and the $\mathrm{FN}$, all $r \mathrm{~s}<.1, p \mathrm{~s}>.40$. 


\section{Discussion}

The present paper examined the effects of cognitive effort and boredom on reward sensitivity, as indexed by the FN. Although in planned contrast analyses we found that participants in both boredom and effort conditions (combined) exhibited a stronger (i.e., more negative) FN response than participants in the control condition, post-hoc analyses and withinsubject multilevel analyses showed that this was primarily driven by participants in the boredom condition. That is, the boredom condition led to greater reward sensitivity than the control condition. Even though previous research has found that people who are generally more prone to experience boredom (i.e., trait boredom) engage in greater reward-seeking and impulsive behaviours, our study was the first to examine the neural consequences of state boredom. Here, we find that boredom, experimentally induced in the laboratory, is associated with heightened brain responses to rewards. This could explain why people who frequently experience boredom act in a more impulsive matter, as sensitivity to reward may lead people to actively seek reward (e.g., Loxton \& Dawe, 2001). Interestingly, despite us providing stimuli that were high in demand (in the effort condition) or absent any demand at all (in the boredom condition), the results make clear that participants found the boredom condition at least somewhat effortful, and more fatiguing than the effort condition. This could suggest that we did not induce pure boredom, or alternatively that the experience of under-stimulation (i.e., boredom) is experienced as subjectively fatiguing. Or, it may be that both effort and boredom lead people to simply be tired. Indeed, it is possible that there might not be such a thing as pure boredom or pure effort. Boredom in the real world can also be mixed with effort (because a task is boring it might take effort to keep paying attention), and even very effortful tasks can be boring/uninteresting. The 
phenomenological experiences of boredom and the effects of boredom on neural representation of reward need to be further investigated.

Although the boredom condition led to greater reward sensitivity than the control condition, the effort condition was neither different from the boredom nor from the control condition. Contrary to predictions, we did not find that participants who engaged in cognitive effort were more reward sensitive than those who did not exert effort; this despite clear evidence that participants in the effort condition reported exerting effort and were (at least to some extent) mentally fatigued. This differs from past research on reward perception, which has found that mental effort leads to increased approach motivation to rewarding stimuli (Schmeichel et al., 2010) and to higher activation in the orbitofrontal cortex (and greater functional connectivity between the orbitofrontal cortex and the inferior frontal gyrus) in response to food cues (Wagner et al., 2013). It is also at odds with recent experimental research on reward seeking, which has shown that depleted individuals will strive for rewards when these are easy (but not difficult) to obtain (Giacomantonio et al., 2014), and that fatigued participants prefer immediate to delayed monetary rewards (Blain et al., 2016). However, the lack of relationship between the effort condition and the FN does fit with research by Gergelyfi and colleagues (2015), who found that neither the motivational value of rewards nor intrinsic motivation varied across time in a twohour-long task that elicited mental fatigue. That is, Gergelyfi and colleagues (2015) did not find that rewards held more motivational value as participants continued to exert mental effort over a prolonged period of time, and we did not find that exerting mental effort led to greater reward sensitivity. Clearly, more research is needed to understand whether effort affects reward processing, and under what conditions this would occur. 
One potential reason why the effects of effort on reward sensitivity may be mixed may have to do with the potential inherent benefits of exerting effort, such that participants who exert effort may feel internally rewarded and not need to look for other (external) sources of rewards for enjoyment or pleasure. Although past research and theorizing have painted effort as inherently aversive (e.g., Kool et al., 2010), others have suggested that effort can have internal rewards such as feelings of competence or self-esteem (Satterthwaite et al. 2012; Gendolla \& Richter, 2010), or inherent interest or enjoyment in the task itself (Cacioppo \& Petty, 1982; see Inzlicht, Shenhav, \& Olivola, in press, for a review). Indeed, previous research has found greater activation in the striatum when participants freely choose a cognitively challenging (rather than an easy) task, reflecting greater intrinsic motivation (Schouppe et al., 2014). Similarly, in our study, it may be that the effort condition was more inherently rewarding than the boredom condition. As far as interest is a reflection of intrinsic motivation (Ryan \& Deci, 2000), we indeed find that the effortful condition was rated as more interesting (i.e., less boring) than the boredom condition. However, we did not find any relation between accuracy in the effort task and the FN ( $r=.15, p=.209)$, suggesting that any internal rewards from being 'better' at the task (as indexed by accuracy) is not reflected in reward sensitivity. Future research is better needed to understand when cognitive effort is viewed as aversive and leads to greater approach/reward sensitivity (as in Schmeichel et al., 2010), and what constitutes an optimal amount of effort (resulting in states of flow; Csikszentmihalyi, 1975, also see Ulrich, Keller, Hoenig, Waller, \& Grön, 2014).

Exploratory supplementary analyses showed that neither effort, boredom, mood, nor selfreported fatigue were related to the $\mathrm{FN}$ for participants in the effort and boredom conditions. Previous analyses have occasionally linked effort reported on a depleting task with ego-depletion 
effects observed in the second task in the sequential task paradigm (Dang, 2016), although these effects tend to be quite small. In contrast, other studies have shown a dissociation between perceptions of effort/fatigue and actual fatigue (e.g., Clarkson et al. 2010). This is in line with other research on phenomenology, especially in the area of emotions. Although phenomenal reports of emotion and emotional behaviour are theoretically assumed to cohere, empirical research rarely finds such evidence. While some studies suggest mild coherence, at least in certain situations (e.g., Mauss, Levenson, McCarter, Wilhelm, \& Gross, 2005), the vast majority of studies find that emotional response systems do not correlate very highly (e.g., Weinstein, Averill, Opton, \& Lazarus, 1968). Such lack of coherence, in fact, has led to a broad rethinking of emotion altogether (Barrett, 2006). This is important because it suggests possible dissociations between people's subjective experience of their affective state and their physiological and behavioral expressions of these states. Future research is thus needed to better understand how phenomenological experiences actually relate to brain and behaviour.

Our results point to the importance of considering the procedures that research on ego depletion uses in the so-called control condition. Indeed, it may be the case that some of the control conditions used in ego depletion studies actually elicit boredom. For example, studies that require participants to read uninteresting texts and cross out letters (e.g., Baumeister et al. 1998), or engage in a repetitive task that does not require mental effort (e.g., Hagger et al. 2016) may all have led to boredom, which we have found to be related with higher subjective fatigue and greater sensitivity to rewards. This may explain the small effects (or lack of effects) found in some depletion studies - the comparison group in some studies may not be a true neutral control. Future studies need to consider the control groups that are used to ensure that boredom is not accidentally induced. 
Despite our initial interest in understanding whether increased fatigue leads to greater enactment of temptation (e.g., Hofmann et al., 2012) and delay discounting (Blain et al., 2016) because of less self-control or because of greater desire, this study did not provide us with adequate information to address this latter possibility. That is, we only assessed how effort and boredom affected subsequent reward processing, but not how they affected subsequent control. Further, since our results indicate that the effort condition was unrelated to reward sensitivity, this would suggest that cognitive effort was unrelated to subsequent experiences of desire. However, given our use of null-hypothesis significance testing, we cannot actually affirm the null (being only able to fail to reject it). Thus, we cannot actually say that cognitive effort had zero effect on reward sensitivity, meaning that our results are somewhat inconclusive.

Additionally, we did not have any indicators of self-control or cognitive control (e.g., a measure of ERN following errors) - future research could include such measures as additional dependent variables.

The current study also furthers research on the FN as an index of reward sensitivity (Proudfit, 2015). Using a standard task for eliciting the FN (Proudfit, 2015), we found again that monetary rewards elicit a larger FN response than positive feedback alone (Weinberg et al., 2014). Adding a new condition with another type of tangible, though non-monetary reward (M\&M candy), results suggested that an appetitive food-type of reward was not different from the neutral (feedback-only) condition. This may have interesting implications for how rewards are processed in the brain, and especially what is rapidly processed in the brain as rewarding. It is interesting that money, which is a social construct, can elicit greater brain reactivity than food, which should arguably be a more readily salient and immediate reward. More research is needed 
to understand whether this is indeed the case (i.e., was it just M\&Ms that were not considered rewarding, or food in general), and why and how such a response would have developed.

\section{Limitations}

This study had several limitations. First, despite the large sample size, we might still have been underpowered, if the effect size was smaller than a medium effect size. For example, we only had $50 \%$ power to detect a small effect size of $\mathrm{f}=.15$. Alternatively, a more powerful manipulation of effort and boredom may have helped us to see more differentiation between the constructs. Second, it is not clear that the boredom condition actually induced pure boredom, particularly since it led to such high experiences of fatigue. Alternatively, the visual-analog scale we used to assess fatigue may have inadvertently picked up on participants' sleepiness (rather than true fatigue), since there were no other options for participants to indicate their levels of sleepiness. That is, maintaining vigilance on a boring task can induce sleepiness, which in turn can be interpreted by participants as fatigue. This may account for the higher subjective fatigue ratings in the boredom condition, and also for a lack of relation between fatigue and reward sensitivity, as sleepiness would not be expected to be related to reward sensitivity.

A third limitation is that we did not have a manipulation check (i.e., measures of fatigue, boredom, etc.) for participants in the control condition; it is thus possible that we had a limited range in the phenomenological measures, which may be why we did not find any relations between phenomenological experiences and reward sensitivity. Additionally, since the control condition involved shorter time-on-task, it may have been qualitatively different than the other two conditions and introduced some unknown carryover effects from participants' previous states (i.e., based on what they were doing and feeling prior to coming to the lab), or by generally reducing the burden on participants. Although in principle we could have looked at the effects of 
time on the $\mathrm{FN}$ to rule them out as a potential reason for the difference between the control condition and the other conditions, we did not have enough trials to get reliable FN waveforms for each block (most blocks had far fewer than the minimum of 10 trials needed for adequate reliability). Future research could address this limitation by either equalizing time-on-task across conditions or controlling for time in the analyses.

Another limitation is that we do not know how much effort participants actually exerted on the two tasks - it may be that some people disengaged from the effortful task (due to difficulty being too high - see Gendolla, Wright \& Richter, 2004), while the boredom task may have led people to exert effort to remain vigilant. This latter possibility is supported by participants' ratings of their effort, which was still over the midpoint even in the boredom task. Additionally, given that we specifically measured effort as task engagement (i.e., "how hard are you trying to do well on the task"), participants in the boredom condition may have discounted any effort they exerted to simply stay on task (i.e., pay attention to the numbers rather than letting their attention wander), which could have further affected their ratings. Despite the effortful condition being more demanding than the boredom condition, perceptions and motivation may have affected the actual use of effort. Indeed, a rich literature on the motivation intensity theory (Brehm \& Self, 1989) describes how multiple processes related to the self (e.g., motivation, ego involvement) can interact with task difficulty to determine the amount of effort that a person will invest in a task (Gendolla \& Richter, 2010). Future research is needed to consider these sources of effort and calibrate both tasks, replicating these results with other tasks requiring cognitive effort (and leading to boredom) to ensure generalizability. Future research can also examine the effects of boredom on subsequent effort engagement (i.e., using a 
sequential task paradigm), and compare these to potential effects of cognitive effort to further understand the consequences of boredom.

A final potential limitation is in the timing of our FN component. Typically the FN is seen between 250 and $350 \mathrm{~ms}$. Our earlier FN is somewhat unusual. However, we used a more objective approach (collapsed localizer) than simple visual inspection of the waveforms to determine our window, the waveform morphology is consistent with studies of the FN, and the component obtained highly dependable estimates with as few as 10 trials. Thus, while early for a typical FN, we feel are measuring the correct ERP component.

\section{Conclusion}

The present study was the first to examine neural sensitivity to rewards following cognitive effort in the general population. Importantly, we contrasted cognitive effort to boredom, an affective state that could be expected to increase sensitivity to rewards. Results suggest that while bored participants were indeed more responsive to rewards than participants in a control condition, this was not the case for participants who had exerted cognitive effort. That is, we found little support for our hypothesis that cognitive effort would increase reward sensitivity. Interestingly, exploratory analyses also found that participants in the boredom condition reported greater subjective fatigue than participants in the cognitive effort condition, suggesting that boredom can be fatiguing. Together, these results shed new light on the phenomenological and cognitive consequences of experiencing boredom. 


\section{References}

Abramson, E.E. \& Stinson, S.G. (1977) Boredom and eating in obese and non-obese individuals. Addictive Behaviors 2(4), 181-185.

Baldwin, S.A., Larson, M.J., \& Clayson, P.E. (2015). The dependability of electrophysiological measurements of performance monitoring in a clinical sample: a generalizability and decision analysis of the ERN and Pe. Psychophysiology, 52(6),790-800.

Barrett, L. F. (2006). Are emotions natural kinds?. Perspectives on psychological science, 1(1), 28-58.

Baumeister, R. F., Bratslavsky, E., Muraven, M., \& Tice, D. M. (1998). Ego depletion: Is the active self a limited resource? Journal of Personality and Social Psychology, 74, 12521265 .

Baumeister, R. F., \& Tierney, J. (2011). Willpower: Rediscovering the greatest human strength. Penguin.

Bench, S. W., \& Lench, H. C. (2013). On the function of boredom. Behavioral Sciences, 3, 459472.

Blain, B., Hollard, G., \& Pessiglione, M. (2016). Neural mechanisms underlying the impact of daylong cognitive work on economic decisions. Proceedings of the National Academy of Sciences, 113(25), 6967-6972.

Blaszczynski, A., McConaghy, N., \& Franknova, A. (1990). Boredom proneness in pathological gambling. Psychological Reports, 67, 35.

Boksem, M. A. S., Meijman, T. F., \& Lorist, M. M. (2005). Effects of mental fatigue on attention: An ERP study. Cognitive Brain Research, 25, 107-116.

Brehm, J. W., \& Self, E. (1989). The intensity of motivation. In M. R. Rozenweig \& L. W. Porter (Eds.), Annual Review of Psychology (pp. 109-131). Palo Alto, CA: Annual Reviews, Inc.

Bress, J. N., \& Hajcak, G. (2013). Self-report and behavioral measures of reward sensitivity predict the feedback negativity. Psychophysiology, 50, 610-616. 
Bruyneel, S. D., Dewitte, S., Franses, P. H., \& Dekimpe, M. G. (2009). I felt low and my purse feels light: Depleting mood regulation attempts affect risk decision making. Journal of Behavioral Decision Making, 22(2), 153-170.

Cacioppo, J. T., \& Petty, R. E. (1982). The need for cognition. Journal of Personality and Social Psychology, 42(1), 116-131.

Carter, E. C., \&Mccullough, M. E. (2014). Publication bias and the limited strength model of self-control: Has the evidence for ego depletion been overestimated. Frontiers in Psychology, 5(823), 1-11.

Clarkson, J. J., Hirt, E. R., Jia, L., \& Alexander, M. B. (2010). When perception is more than reality: the effects of perceived versus actual resource depletion on self-regulatory behavior. Journal of Personality and Social Psychology, 98(1), 29-46.

Clayson, P. E., Baldwin, S. A., \& Larson, M. J. (2013). How does noise affect amplitude and latency measurement of event-related potentials (ERPs)? A methodological critique and simulation study. Psychophysiology, 50, 174-186.

Clayson P.E., \& Miller, G.A. (in press). ERP Reliability Analysis (ERA) Toolbox: An opensource toolbox for analyzing the reliability of event-related brain potentials. International Journal of Psychophysiology.

Csikszentmihalyi, M. (1975). Play and intrinsic rewards. Journal of humanistic psychology.

Dang, J. (2016). Commentary: A Multilab Preregistered Replication of the Ego-Depletion Effect. Frontiers in Psychology, 7.

Dien, J. (2010). The ERP PCA Toolkit: An open source program for advanced statistical analysis of event-related potential data. Journal of Neuroscience Methods, 187, 138-145.

Eastwood, J. D., Frischen, A., Fenske, M. J., \& Smilek, D. (2012). The unengaged mind defining boredom in terms of attention. Perspectives on Psychological Science, 7(5), 482-495.

Friese, M., Loschelder, D.D., Gieseler, K., Frankenbach, J., \& Inzlicht, M. (in press). Is ego depletion real? An analysis of arguments. Personality and Social Psychology Review.

Gailliot, M. T., Baumeister, R. F., DeWall, C. N., Maner, J. K., Plant, E. A., Tice, D. M., ... \& Schmeichel, B. J. (2007). Self-control relies on glucose as a limited energy source: 
willpower is more than a metaphor. Journal of personality and social psychology, 92(2), 325.

Garrison, K. E., Crowell, A. L., Finley, A. J., \& Schmeichel, B. J. (2017). Effects of prior mental effort on picture processing: An ERP investigation. Psychophysiology.

Gendolla, G. H., \& Richter, M. (2010). Effort mobilization when the self is involved: Some lessons from the cardiovascular system. Review of General Psychology, 14(3), 212.

Gendolla, G. H. E., Wright, R., \& Richter, M. (2012). Effort intensity: Some insights from the cardiovascular system. In R. Ryan (Ed.), The Oxford handbook of human motivation (pp. 420-438). Oxford, UK: Oxford University Press.

Gergelyfi, M., Jacob, B., Olivier, E., \& Zénon, A. (2015). Dissociation between mental fatigue and motivational state during prolonged mental activity. Frontiers in behavioral neuroscience, 9, 176.

Giacomantonio, M., Jordan, J., Fennis, B. M., \& Panno, A. (2014). When the motivational consequences of ego depletion collide: Conservation dominates over rewardseeking. Journal of Experimental Social Psychology, 55, 217-220.

Goldberg, Y. K., Eastwood, J. D., LaGuardia, J., \& Danckert, J. (2011). Boredom: An emotional experience distinct from apathy, anhedonia, or depression. Journal of Social and Clinical Psychology, 30(6), 647.

Hagger M. S., Chatzisarantis N. L. D., Alberts H., Anggono C. O., Batailler C., Birt A. R., .. . Zwienenberg M. (2016). A multilab preregistered replication of the ego-depletion effect. Perspectives on Psychological Science, 11, 546-573.

Hagger, M. S., Wood, C., Stiff, C., \& Chatzisarantis, N. L. D. (2010). Ego depletion and the strength model of self-control: a meta-analysis. Psychological Bulletin, 136(4), 495-525.

Hockey, R. (2013). The psychology of fatigue: Work, effort and control. Cambridge University Press.

Hofmann, W., Vohs, K. D., \& Baumeister, R. F. (2012). What people desire, feel conflicted about, and try to resist in everyday life. Psychological Science, 23(6), 582-588. 
Hopstaken, J. F., van der Linden, D., Bakker, A. B., Kompier, M. A., \& Leung, Y. K. (2016).

Shifts in attention during mental fatigue: Evidence from subjective, behavioral, physiological, and eyetracking data. Journal of Experimental Psychology: Human Perception and Performance, 42, 878-889.

Inzlicht, M., \& Berkman, E. T. (2015). Six questions for the resource model of control (and some answers). Social and Personality Psychology Compass.

Inzlicht, M., Gervais, W., \& Berkman, E. (2015). Bias-Correction Techniques Alone Cannot Determine Whether Ego Depletion is Different from Zero: Commentary on Carter, Kofler, Forster, \& McCullough, 2015. Available at SSRN: http://ssrn.com/abstract $=2659409$ or http://dx.doi.org/10.2139/ssrn.2659409

Inzlicht, M., Schmeichel, B. J., \& Macrae, C. N. (2014). Why self-control seems (but may not be) limited. Trends in cognitive sciences, 18(3), 127-133.

Inzlicht, M, Shenhav, A., \& Olivola, C.Y. (in press). The effort paradox: Effort is both costly and valued. Trends in Cognitive Sciences. doi: 10.1016/j.tics.2018.01.007

Iso-Ahola, S.E., Crowley E.D. (1991). Adolescent substance abuse and leisure boredom. Journal of Leisure Research. 23(3):260-271.

Kahneman, D. (2011). Thinking, fast and slow. Macmillan.

Kato, Y., Endo, H., \& Kizuka, T. (2009). Mental fatigue and impaired response processes: Event-related brain potentials a Go/NoGo task. International Journal of Psychophysiology, 72, 204-211.

Kool, W., McGuire, J.T., Rosen, Z.B., \& Botvinick, M.M. (2010). Decision making and the avoidance of cognitive demand. J. Exp. Psychol.: Gen. 139:665-82

Kurzban, R. (2010). Does the brain consume additional glucose during self-control tasks? Evolutionary Psychology : An International Journal of Evolutionary Approaches to Psychology and Behavior, 8(2), 244-59.

Kurzban, R., Duckworth, A., Kable, J. W., \& Myers, J. (2013). An opportunity cost model of subjective effort and task performance. The Behavioral and Brain Sciences, 36(6), 661-79. 
Larson, M.J., Fair J.E., Good, D.A. \&, Baldwin, S.A.(2010). Empathy and error processing. Psychophysiology, 47(3), 415-24.

Loxton, N. J., \& Dawe, S. (2001). Alcohol abuse and dysfunctional eating in adolescent girls: the influence of individual differences in sensitivity to reward and punishment. International Journal of Eating Disorders, 29, 455-462.

Luck, S. J., \& Gaspelin, N. (2016). How to get statistically significant effects in any ERP experiment (and why you shouldn't). Psychophysiology.

Mackworth, N. H. (1948). The breakdown of vigilance durning prolonged visual search. Quarterly Journal of Experimental Psychology, 1(1), 6-21.

Mauss, I. B., Levenson, R. W., McCarter, L., Wilhelm, F. H., \& Gross, J. J. (2005). The tie that binds? Coherence among emotion experience, behavior, and physiology. Emotion, 5(2), 175.

Milyavskaya, M.,\& Inzlicht, M. (2017). Attentional and motivational mechanisms of selfcontrol. In D. de Ridder, M. Adriaanse, and K. Fujita (Eds). Handbook of Self-Control in Health \& Well-Being. Routledge.

Myhre, S. K., Crockett, A. C., \& Rokke, P. D. (2015). Boredom Proneness and Emotion Regulation Predict Emotional Eating Behaviors. Journal of Health Psychology, 20, 670680.

Pattyn, N., Neyt, X., Henderickx, D., \& Soetens, E. (2008). Psychophysiological investigation of vigilance decrement: Boredom or cognitive fatigue? Physiology \& Behavior, 93, 369-378.

Proudfit, G. H. (2015). The reward positivity: From basic research on reward to a biomarker for depression. Psychophysiology, 52(4), 449-459.

Ryan, R. M., \& Deci, E. L. (2000). Self-determination theory and the facilitation of intrinsic motivation, social development, and well-being. American psychologist, 55(1), 68.

Satterthwaite, T. D., Ruparel, K., Loughead, J., Elliott, M. A., Gerraty, R. T., Calkins, M. E., ... \& Wolf, D. H. (2012). Being right is its own reward: load and performance related ventral striatum activation to correct responses during a working memory task in youth. Neuroimage, 61(3), 723-729. 
Schmeichel, B. J., Harmon-Jones, C., Harmon-Jones, E. (2010). Exercising self-control increases approach motivation. Journal of Personality and Social Psychology, 99, 162-73.

Shenhav, A., Musslick, S., Lieder, F., Kool, W., Griffiths, T. L., Cohen, J. D., \& Botvinick, M. M. (2017). Toward a rational and mechanistic account of mental effort. Annual review of neuroscience, 40, 99-124.

Shmueli, D., \& Prochaska, J. J. (2009). Resisting tempting foods and smoking behavior: Implications from a self-control theory perspective. Health Psychology, 28, 300-306.

Threadgill, A. H., \& Gable, P. A. (2016). Approach-motivated pregoal states enhance the reward positivity. Psychophysiology.

Ulrich, M., Keller, J., Hoenig, K., Waller, C., \& Grön, G. (2014). Neural correlates of experimentally induced flow experiences. Neuroimage, 86, 194-202.

Vohs, K. D., \& Faber, R. J. (2007). Spent resources: Self-regulatory resource availability affects impulse buying. Journal of Consumer Research, 33, 537-547

Vohs, K. D., \& Heatherton, T. F. (2000). Self-regulatory failure: A resource-depletion approach. Psychological Science, 11, 249-254.

Wagner, D. D., Altman, M., Boswell, R. G., Kelley, W. M., \& Heatherton, T. F. (2013). Selfregulatory depletion enhances neural responses to rewards and impairs top-down control. Psychological science, 24, 2262-2271.

Weinberg, A., Riesel, A., \& Proudfit, G. H. (2014). Show me the money: the impact of actual rewards and losses on the feedback negativity. Brain and Cognition, 87, 134-139.

Weinstein, J., Averill, J. R., Opton Jr, E. M., \& Lazarus, R. S. (1968). Defensive style and discrepancy between self-report and physiological indexes of stress. Journal of Personality and Social Psychology, 10(4), 406.

Westbrook, A., \& Braver, T. S. (2015). Cognitive effort: A neuroeconomic approach. Cognitive, Affective, \& Behavioral Neuroscience, 15(2), 395-415.

Westbrook, A., Kester, D., \& Braver T.S. (2013). What is the subjective cost of cognitive effort? Load, trait, and aging effects revealed by economic preference. PLOS ONE 8:e68210 
Wuensch, (2012). Using SPSS to Obtain a Confidence Interval for R2 From Regression.

Instructions and SPSS script retrieved from http://core.ecu.edu/psyc/wuenschk/SPSS/CIR2-SPSS.zip. 